\title{
Data flow for object manufacturing inside of information system for Industry 4.0
}

\author{
Katarina Monkova ${ }^{1}$, Peter Monka ${ }^{2}$, Miorita Ungureanu ${ }^{3}$, Nicolae Ungureanu ${ }^{4}$, Oleksandr \\ Gusak $^{5}$, Milan Edl ${ }^{6}$ \\ \{katarina.monkova@tuke.sk ${ }^{1}$, peter.pavol.monka@tuke.sk ${ }^{2}$, \\ miorita.ungureanu@cunbm.utcluj.ro $\left.{ }^{3}\right\}$
}

FMT TU Kosice with the seat in Presov, Sturova 31, 08001 Presov, Slovakia ${ }^{1.2}$, Technical University of Cluj-Napoca, Department of Engineering and Technologic Management, Baia Mare, Romania ${ }^{3,4}$, Faculty of Technical Systems and Energy Efficient Technologies, Sumy State University, Ukraine ${ }^{5}$,

Faculty of Mechanical Engineering, University of West Bohemia, Pilsen, Czech Republic ${ }^{6}$

\begin{abstract}
Most of today's information systems are not fully integrated. Companies, suppliers, and customers are rarely closely linked. Functions within a chain from the enterprise to the shop floor level are not fully integrated. Even in the engineering itself lacks complete integration - from products to plants to automation. With Industry 4.0, companies, departments, functions, and capabilities will become much more cohesive, as cross-company, universal data-integration networks evolve and enable truly automated value chains. The article deals with specification of data flow related to an object manufacturing inside of information system for Industry 4.0. There are described basic characteristics of both data flow in this paper. An older one, which was used in the past, but it can be found also in current practice, as well as new approach to data flow using STEP NC (a machine tool control language). A reliable information system is one of the necessities to be the data flow in a company continuous. Due to this reason, authors designed software application based on concept Industry 4.0 that should help to improve productivity and labour efficiency in the company. Basic principles of object coding system that are included inside newly developed information system, are elaborated in the next part of the article.
\end{abstract}

Keywords: data flow, information system, Industry 4.0, coding

\section{Introduction}

One of the most closely watched indicators of long-term economic prospects is productivity. Rising productivity is the key making possible permanent increases in the standard of living. Changes in a technology are the only source of permanent increases in productivity, but a number of transient factors can affect both true and "measured" productivity. [1]

The present situation in the industry is characterized as a period of intense progress of technologies at the significant computer aid in all branches of industry. In connection with the technical progress it is increasing the pressure on the manufacturers to develop and make the products as soon as possible at the minimal cost in required quality. The product has to be competitive, it has to be up to qualitative and functional standard, it has to have reasonable price, efficacious design, and it has to consider safety, ergonomic and other aspects, which decided about its marketability. All the information represents the know-how of the plant, so it is very 
important to process data within reliable information system. That is why the system has to ensure $[2,3]$

- production effectivity and quality increasing,

- to be its structure dynamically adaptable for actual situation and for user specific conditions with minimum negative effects,

- selection in which way will be a process plan created, whether approach of group technology will be used or whether it will be done for every part separately; If the part will be manufactured using of NC (Numerical Control) machine, the producer will be able to decide how the NC program originate, whether it will be written manually or whether it will be created by means of CAM (Computer Aided Manufacturing) system;

- selection of parts with the similar material and dimensional characteristics, with similar process plans; therefore, it will be considerably able to save the batch time;

- to be parameters processed easier and faster,

- to be preparatory time for a technological documentation reduced;

- to be information used not only for technological documentation generation, but also for details processing due to data storage, for economical needs and for wage records.

The basic data flow in production today show Fig. 1.

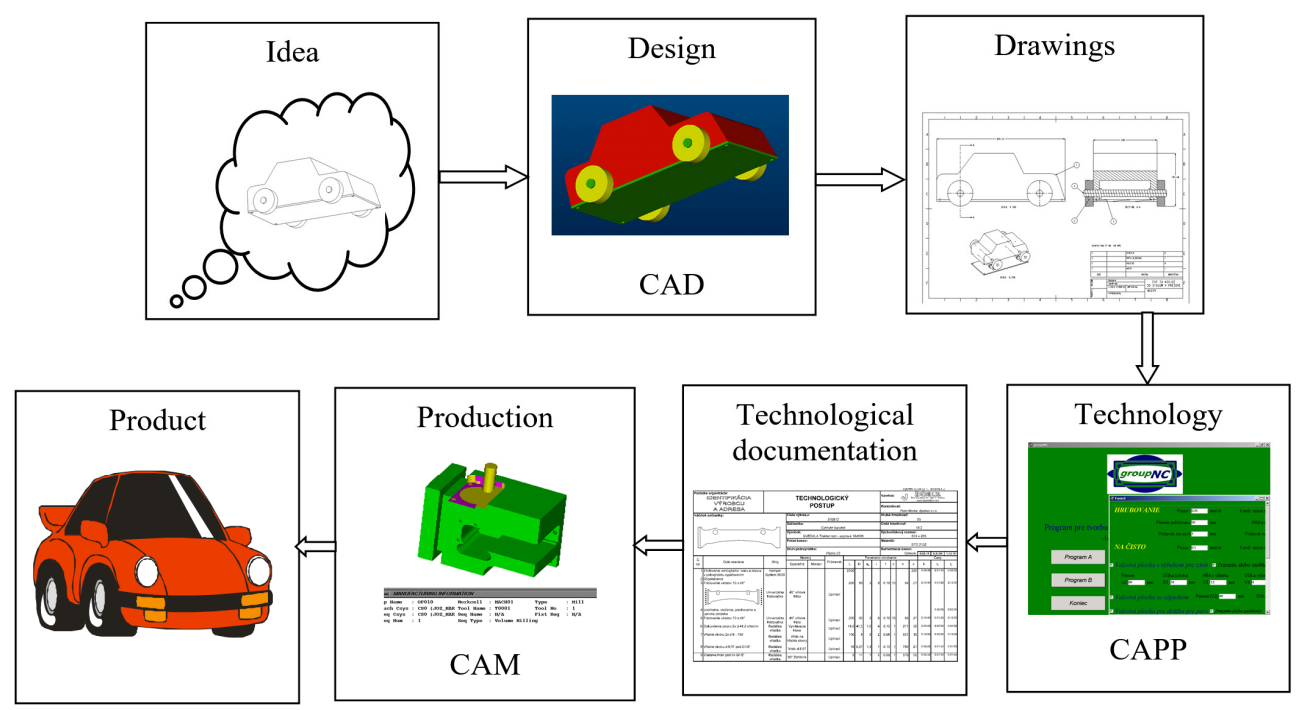

Fig. 1. Basic data flow in production today

One of the manners how to simplify the data flow and data translation at the object manufacturing is the introducing of STEP NC (a machine tool control language) philosophy into production. In this article, the comparison of the data flow and data transferring without and with the using of STEP NC will be done, including the code designing that respects the necessity of new approach. 


\section{Specification of data flow related to the object manufacturing}

\subsection{Data flow in the past and today}

Typically, three different software applications have been used for a part manufacturing. They are: CAD (Computer Aided Design) software to make the design of the part, CAM software to calculate the tool paths based on the design, compensating for the cutter's geometry, adding spindle commands, and Control software to read the tool path and let the machine actually move along these paths. Communication between these three programs is done by means of special files. File translation is a process in which one data format is converted into another. Data flow could be divided into next several steps (Fig.2): [4]

- Primary information about a product is imported into the CAM system. Usually 3D CAD model is imported.

- In mechanical engineering CAM is used to calculate toolpaths to cut material. The CNC programmer just specifies the machining operations and the CAM system creates the toolpath, usually written in CL data (Cutter Location data) file.

- Calculated toolpath is imported to the postprocessor which converts the CL data to the NC program - the specific machine codes that are required to operate numerically controlled machine tools. Machine codes vary by machine tool. The output from a postprocessor should be usable in the controller without further modification.

- NC program written in a notation called G-code is exported to the NC machine and the manufacturing process can begin.

Digital product data have to contain sufficient information to cover a product's entire life cycle, spanning design, analysis, manufacture, quality control testing, inspection and product support functions. The present situation in production data flow is shown in Fig. 2.

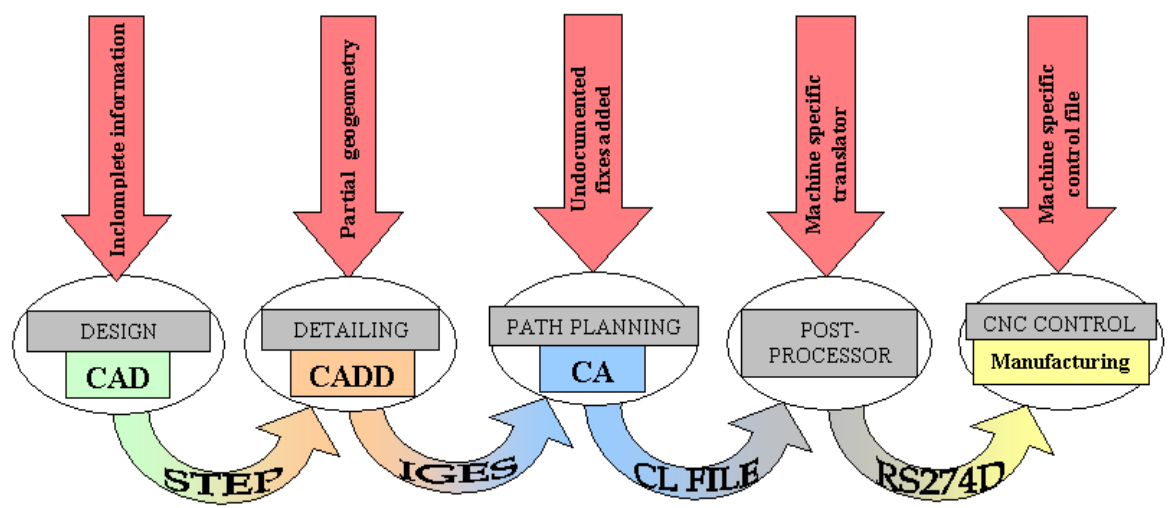

Fig. 2. Data flow without using of STEP NC

Design creates the specification for a product as a 3D model. Detailing decides the manufacturing requirements for the product by making a drawing. Path planning generates tools paths. Manufacturing controls production. The job of design is performed using a CAD system, the job of detailing is performed using a drawing CADD (Computer Aided Design Drafting) system, the job of path planning is performed using a CAM (Computer Aided Manufacturing) system, and job of manufacturing is controlled using a CNC (Computer Numerical Control) 
system. In many cases the CAD, CADD and CAM functions are combined into a single integrated $\mathrm{CAD} / \mathrm{CAM}$ system but in all cases the $\mathrm{CNC}$ function is performed by a separate system. $[5,6]$

In the data flow introduced in Fig. 2, an information can be lost, because incomplete data is sent from the CAD to the CAM The fixes to the geometry are made in the CAM and there is no communication back to the $\mathrm{CAD}$, because only the surface data are communicated to the postprocessor. It is due to the RS274D standard only allows axis movement data to be communicated to the control. This means that no adjustments can be made on the control in response to changes in the available tooling, the control cannot optimize the machining process for the capabilities of the selected machine, and the operator cannot rely on software in the control to check the safety of the set-up and the program.

One of the most important links in the $\mathrm{CNC}$ machining process is postprocessor. The company can have best programming system, the best DNC system, and the most accurate machines, but without dependable postprocessors the code getting to its machine could cause problems. Most machines require some tweaking to the postprocessor to make it to produce code to user's likings and programming habits. Most CAM software comes with built in postprocessor; however, there are many stand-alone postprocessors. It is the last software link between an ideal CAD model and a 'real' machined part.

\subsection{Future data flow considered by the information system}

Data flow in the future, that is also considered in the newly designed information system, is based on the concept using STEP NC. It enables a product model database to serve as direct input to a CNC machine tool. No separate files of tool paths. No G or M codes. No post processors. This is a radically different approach to CNC programming. STEP NC is an extension to STEP, the STandard for the Exchange of Product model data. [7]

STEP is the international standard that specifies a neutral data format for digital information about a product. STEP allows this data to be shared and exchanged among different and otherwise incompatible computer platforms. STEP NC standardizes how information about CNC machining can be added to parts represented in the STEP product model. [8]

STEP NC defines a CNC part program as a series of operations that remove material defined by features. The features supported include holes, slots, pockets and volumes defined by $3 \mathrm{D}$ surfaces. Each operation contributes to the manufacture of a feature by defining the volume of material to be removed, the tolerances, the type of tool required and some basic characteristics such as whether this is a roughing or finishing operation. The operations are then sequenced into a work plan that converts the stock into the final part. The work plan may be sophisticated and include conditional operations that depend on the results of probing operations, and it may be divided into sub-plans to be executed concurrently on machines that have multiple cutting heads.

STEP NC has the Application Protocol number AP-238 within the STEP framework. A key feature of STEP NC, AP-238 programs, is that they are machine and organization independent. If a machine has the underlying capabilities (axes, table size etc), then a STEP NC "compiler" should be able convert the part program into a sequence of tool movements for that machine. If a CNC has a Tool Cutter Programming (TCP) interface then the tool movements can be executed directly without converting to axis movements. This has significant consequences for industry. In the new method, the enterprises are able to continue in using their existing systems for CAD, CADD and CAM, but the final result is sent to the CNC as a STEP NC AP-238 file instead of an RS274D file. The modified data flow with the using of STEP NC is shown in Fig. 3. [9-11] 
STEP NC allows a complete database of machining information to be built around the digital product model and ultimately makes it possible for this enhanced product model to serve as machine tool input. This database is structured such that part features are linked to specific "working steps," generic descriptions of various machining operations. STEP NC working steps are roughly equivalent to machining commands formatted as traditional M and G codes. With the concept of "working steps" in place, the manufacturing process becomes streamlined. Now, a machine tool can receive a file with STEP NC data, "know" what it means, and proceed milling the piece without any more instructions. There will be no more programming the machine tool for each individual piece. Moreover, with a set of standards "working steps" in place, all manufacturers will be able to share information reliably and instantaneously. [12]

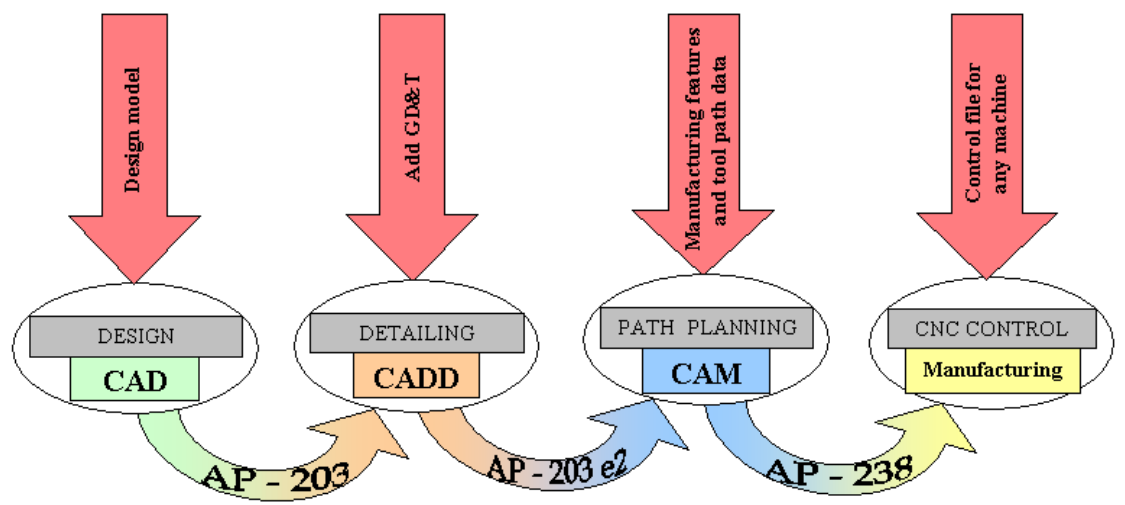

Fig. 3. Data flow using STEP NC

\section{Object coding inside information system}

The objects in machine engineering such as parts, machines, equipment and other issues are possible to be modelled on various stages and with various goals. It will result in various types of models (physical, simulation, computer, mathematical and other). Every of these objects it is possible to consider as system, which consists of other features, respectively as a feature that is part of some system. To be the objects implemented inside an information system and to be possible to work with them, it is advantageous to encode every object with a code, which would express all its characteristics. [13]

In regard to a large number of parameters that are variable in consequence of the varied manufacturing process conditions, it is most suitable to use the type of code at which are the starting positions reserved for the characteristic properties of the object. Other positions are attached to the attribute part of code according to the need to define the classification of the object. On the basis of this structure it is possible the manufacturing system consider to be set, which is unification of subsets marked as subsystems. Following objects have been coded inside newly developed information system: manufactured articles (components, assemblies, ...) within a set with name SEGMENT, used technological operations within a set named OPERATION and used technical devices within a set named EQUIPMENT.

The codes and requirements for newly developed information system introduced bellow represent only a part of complex system. 


\subsection{Subsystem Segment}

The basis of subsystem "Segment" is the classification code for the segment description, which represents the start point of whole system. The suggested coding system keeps the space for the process plans creating not only for cutting technology but also for other technologies. The codes cover the following characteristics:

- geometrical shape,

- class of part,

- manufacturing characteristics,

- $\quad$ class of dimensions.

The example of the selected surface coding system with the possibility to manufacture surfaces by individual technological operations is shown on the Fig. 4 . Numbers 0 or 1 describes the true or false of this manufacturability.

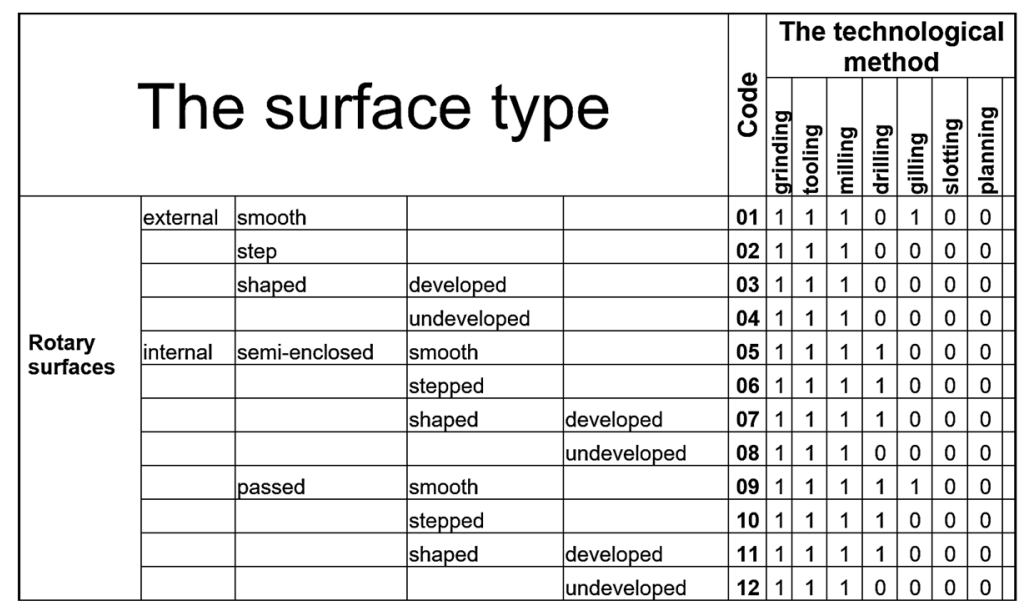

Fig. 4. The example of the surface coding with the possibility of manufacturability by individual technological operations

During the creation of software application, it was suggested several manners of the segment classification, for example according to the types of surfaces that didn't comply from the view of the classification complexity. An example of generated code structure is shown in Fig. 5.

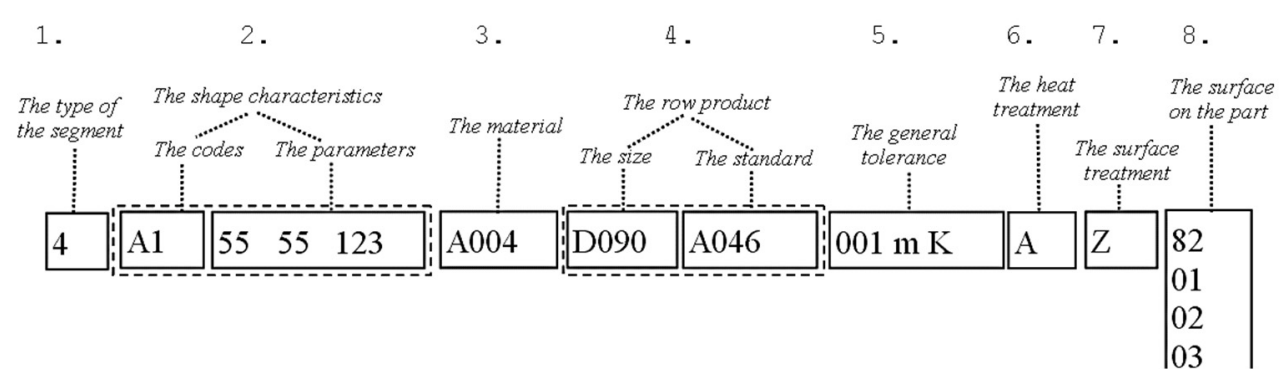

Fig. 5. The structure of individual code parts in subsystem Segment 
The coding of the Segment in this software application go out from the assumption that the data registered in this module will be next used at the creation of technological or drawing documentation and the parameters already once defined will be possible to record by another database module. Therefore, the Segment code can to appear too difficult on the first sight, but at the work in user interface it is its creating very simply and it is aids by already partially charged by data bank.

The background, where the data are loaded and processed, is displayed in the Fig.6. It is necessary to say that it is important to verify the functionality of every NC programs in a practice and consequently the program to debug. The same holds in the case of NC programs prepared in the software conditions.

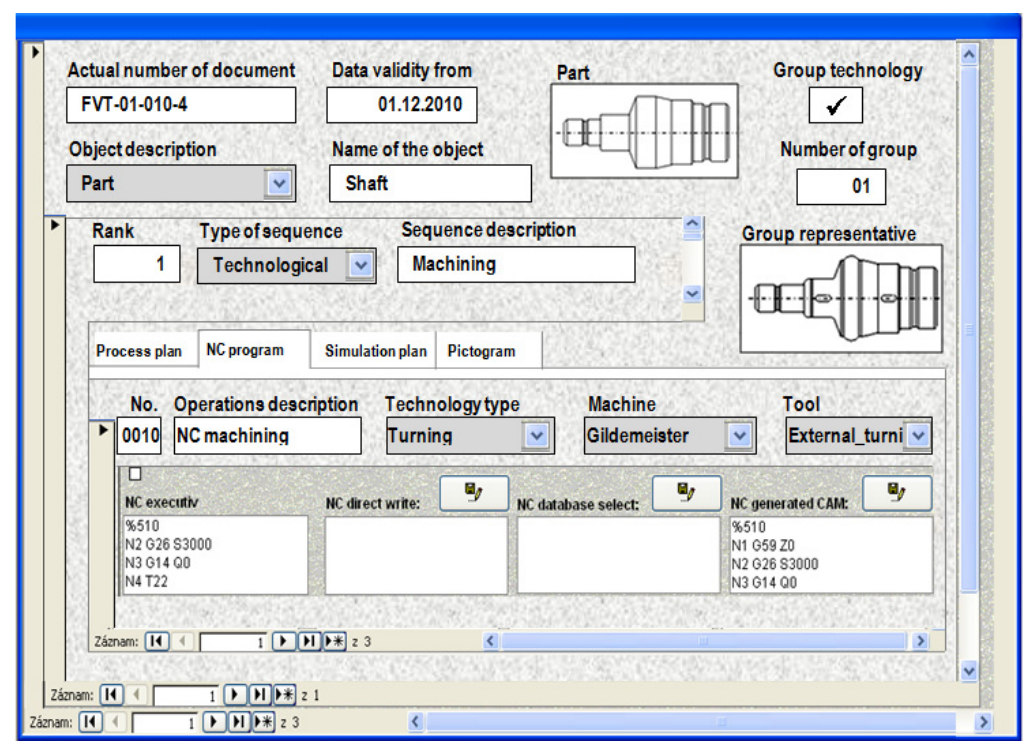

Fig. 6. Environment Software application for NC program loading

\subsection{Structure of operation subsystem}

On the basis of suitable code definition for the Structure of operation subsystem it is possible to determinate three stages:

- The class of the machining,

- The type of the machining,

- The process of the machining.

It is possible to specify the concrete machining operation by means of these three stages. The example of the Structure of operation coding is shown in Fig. 7, which shows the code meaning.

Inside the created software application exist also other technologies and so this subsystem is expanded to the stages:

- technology,

- technological grade,

- technological type,

- technological process. 


\begin{tabular}{|c|c|c|c|}
\hline \multicolumn{2}{|c|}{$\begin{array}{l}\text { 1.code digit } \\
\text { The class of the } \\
\text { machining }\end{array}$} & $\begin{array}{l}\text { 2.code digit } \\
\text { The type of the } \\
\text { machining }\end{array}$ & $\begin{array}{l}\text { 3.code digit } \\
\text { The process of the } \\
\text { machining }\end{array}$ \\
\hline 0 & rotary machining & autom. lathe work without thread & thread cutting internal \\
\hline 1 & facing & lathe work without thread-cutting & thread cutting external \\
\hline 2 & drilling and boring & grinding & thread milling internal \\
\hline 3 & manual work, generally & treading & thread milling external \\
\hline 4 & surface treatment & autom lathe work with thread & thread rolling internal \\
\hline 5 & inspection, checking & lathe work with thread & thread rolling external \\
\hline 6 & heat treatment & gear cutting & thread grinding internal \\
\hline 7 & non-cutting processes & vacant & thread grinding external \\
\hline 8 & metal joining & vacant & worm machining \\
\hline 9 & casting, etc. & vacant & other \\
\hline
\end{tabular}

Fig. 7. The example of the Structure of operation coding

\subsection{Subsystem Equipment}

The term "Equipment" is, in this case, used for the cover of wide spectrum of various product equipment, such as:

- $\quad$ production spaces (the halls, workshops, ...)

- $\quad$ equipment for the energy production and energy distribution

- machining equipment

o tools

$\circ$ jigs and fixtures

- machines

○ machining equipment

- equipment for the workshops of manual operations

o other

- $\quad$ measuring and testing equipment

- $\quad$ conveying devices

- $\quad$ equipment for storage

- $\quad$ other devices (for example computer techniques, ...)

The subsystem „Equipment” represents very large, rugged and heterogeneous structure of individual objects. Therefore, it was used at the suggestion of the coding the hybrid type of code. An example of the machining equipment dividing can be following:

\section{Machines}

Machines can be divided from various aspects; the most advantageous one is the classification on the basis of used technology. In this case we can speak e.g. about the machines for:

- Machining,

- Moulding,

- Casting,

- Welding,

- Assembly and other.

Next classification can be based on the type of machining:

I. The turning machines,

II. The milling machines, 
III. The drilling and boring machines,

IV. The centres of machining and other.

Tools

The tools are very important part of production process. To be tools coded exactly, it is necessary to consider following parameters:

- technological operation that the tools are able to execute,

- technological and geometrical limitation,

- maximal and minimal values of the working parameters,

- type of the work holding,

- environment, which can be tools used in.

Jigs and fixtures

It is needed to consider these issues at the coding of jigs and fixtures:

- The devices, which can be used on,

- The maximal and minimal values of the working parameters,

- The environment, which can be jigs and fixtures used in.

\section{The accessory equipment}

The accessory equipment is often essential and necessary for the flow of some operation. It was possible to choose the hybrid type of code in regard to the ambiguity of its using definition (e.g. the same medium can be sometimes used as the cooling mixture and some other time as oil).

\section{Conclusions}

$\mathrm{NC}$ machines need the $\mathrm{NC}$ program for the running today. It can be written „manually "by direct writing of $\mathrm{NC}$ instructions, or automatically by means of postprocessor. To handle the wide variety of RS274D dialects (over 5,000), postprocessors are used to automatically convert tool path data generated by CAM to G-codes specific for CNC's RS274D dialect. In some cases, this is done by the CAM system internally, in others the CAM system generates APT "cutter location" data that is converted to G-codes by the postprocessor.

Postprocessing is not a value-added step, therefore the CNC will not be needed to use Gcodes in the future. There will be no need for creating a new and separate file of tool path data. Tool paths will be figured out in the CNC itself, based on the product model. That means there's no need for postprocessors either. Data will be formatted for execution by the machine within the CNC. And because the product model won't change, it will be available for machining "hard copies" whenever and wherever needed. Postprocessors will no longer be of concern to endusers. If they exist at all they will be buried inside the control as a way to translate the working steps in a STEP-NC program into the instruction codes understood by the CNC. No one will need to see these codes except under special circumstances just like no one typically sees the assembler code of a computer anymore. [14,15]

The requirements for post processors eliminate STEP-NC, because STEP-NC has no dialects. It allows building a complete database of machining information around it. Manufacturing organizations need to be able to seamlessly share information over the Internet. Using STEP-NC, machine shops can reduce "set up" times by up to $35 \%$ if they can seamlessly read the 3D product geometry and manufacturing instructions of their customers, and original equipment manufacturers can reduce the time they spend preparing data for their suppliers up to $75 \%$ if they can seamlessly share the design and manufacturing data in their databases. [16] 
The designing new philosophy and the development of new software product for the creation of multivariant process plans is the intent of concept Industry 4.0. This approach enables to increase of effectivity already at the beginning of product design and to improve the process of technological documentation creation without of the influence on its complexity. Generated application will be built by modular manner to allow flexible adapt data structure to user specific conditions and to satisfy the specification of simple implementation into already existing information structure of the plant. The output data of the system will be able to utilize not only for the generating of technological documentation but also to the processing of details for manufacturing, store, economic and wage records, thereinafter for the creating and archiving of $\mathrm{NC}$ programs and for the data registration, too. It is assumed the practical verification of the final product in real conditions of manufacturing plants

Acknowledgments. The present contribution has been prepared with direct support of Ministry of Education, Science, Research and Sport of Slovak Republic through the projects KEGA 007TUKE-4/2018 and VEGA 1/0795/19.

\section{References}

[1] Bekes, J., Andonov, I.: Analysis and synthesis of machining objects and processes, ALFA, Bratislava, ISBN 60-080-86 (1986)

[2] Gawronska, E. \& Sczygio, N., 2010. Application of mixed time partitioning methods to raise the efficiency of solidification modeling, 12th Intemational Symposium on Symbolic and Numeric Algorithms for Scientific Computing, IEEE, 99-103.

[3] Vychytil, J. \& Holecek, M, 2010. The simple model of cell prestress maintained by cell incompressibility, Mathematics and computers in simulation, 80(6), 1337-1344.

[4] Durmuş, K. et al.: Integrated Knowledge-Based System for Machine Design, Advances in Mechanical Engineering, Vol. 2013 (2013)

[5] Kishinami, T.: XML-based High Level NC data Modelling for Manufacturing Data Management. ISO TC 184/SC4/WG3, Charleston (2000/10/19)

[6] Lapcik, L. et al., 2016. Study of bread staling by means of vibro-acoustic, tensile and thermal analysis technique. J. of Food Eng., 178 (6) 31-38.

[7] Turisova, R. et al., 2012. Verification of the risk assessment model through an expert judgment, Quality innovation prosperity, 16(1), 37-48.

[8] International standard, Data model for Computerized Numerical Controllers, ISO 14649-1, Part 10: General Process Data

[9] Kadarova, J. et al., Proposal of performance assessment by integration of two management tools, Quality Innovation Prosperity 17(1), 2013, pp. 88-103.

[10] Stojadinovic, S.M., Majstorovic, V.D., Developing engineering ontology for domain coordinate metrology, In. FME Transactions, vol. 42(3), 2014, pp. 249-255.

[11] Vuković, A., Perinić, M., Ikonić, M.: Conceptual Framework for Creating Customized modular CAPP system, Eng. Rev. 31-1, 35-43 (2011)

[12] Monka, P. et al.: Multivariant process plans design in relation to the European market, DAAAM Symposium, Trnava, Slovakia, pp. 901-902 (2008)

[13] Zetek, M., Zetkova, I.: Increasing of the cutting tool efficiency from tool steel by using fluidization method, 25th DAAAM Int. Symposium, Vienna, Vol. 100, pp. 912-917, (2015)

[14] R. Cep et al.: Surface roughness after machining and influence of feed rate on process, Key Engineering Materials, Vol. 581, pp. 341-347, (2014)

[15] Monkova, K. et al.: Newly developed software application for multiple access process planning, Advances in Mechanical Engineering, pp. 39071-39071, (2014)

[16] Jurko, J. et al.: Verification of cutting zone machinability during the turning of a new austenitic stainless steel, Adv. Comp. Sci. Edu. Appl., 202/2, pp. 338-345, (2011) 